\title{
Implementation of a quality control for radio occultation observations in the presence of large gradients of atmospheric refractivity
}

\author{
L. Cucurull ${ }^{1,2}$ \\ ${ }^{1}$ NOAA ESRL Global Systems Division, Boulder, CO, USA \\ ${ }^{2}$ Cooperative Institute for Research in Environmental Sciences, Boulder, CO, USA
}

Correspondence to: L. Cucurull (lidia.cucurull@noaa.gov)

Received: 15 September 2014 - Published in Atmos. Meas. Tech. Discuss.: 15 October 2014

Revised: 10 February 2015 - Accepted: 12 February 2015 - Published: 16 March 2015

\begin{abstract}
In preparation for the launch of the first six satellites of the COSMIC-2 mission in equatorial orbit, and the larger number of observations that such a mission will provide in the lower tropical troposphere, work is underway at the National Oceanic and Atmospheric Administration (NOAA) to improve the assimilation of radio occultation (RO) observations, particularly in the lower tropical troposphere. As part of the improvement of the bending angle forward operator at the National Centers for Environmental Prediction (NCEP), additional quality controls aimed to detect and reject observations that might have been affected by super-refraction conditions have been implemented and tested. The updated quality control procedures also address the situation where the model detects atmospheric superrefraction conditions. This paper describes the limitations of the current standard quality controls and discusses the implementation of additional quality control procedures to address the limitations of assimilating observations likely affected by the super-refraction conditions, either in the model simulation or in the retrieval process.
\end{abstract}

\section{Introduction}

The planetary boundary layer (PBL) extends from the surface up to a height that ranges anywhere from a few tens of meters to several kilometers. The PBL is directly influenced by the presence of the Earth's surface, responding to forcing such as frictional drag, solar heating, and evapotranspiration. A realistic representation of the PBL in weather and climate models is necessary, since it is within this layer that the exchange of energy, momentum, and mass between the earth's surface and the free troposphere takes place (Heckley, 1985; Albrecht et al., 1986; Betts and Ridgeway, 1989). While global characterization of the PBL over land has been investigated extensively with the use of conventional observations (Lettau and Davidson, 1957; Swinbank, 1968; Izumi, 1971; Clarke and Brook, 1979), the amount of observations available in the marine boundary layer (MBL) is rather scarce. (The MBL is the PBL over the ocean.) Representation of the MBL through traditional remote sensing has some well-known limitations due to the presence of clouds and/or limited vertical resolution. However, Global Positioning System (GPS) radio occultation (RO) limb soundings can penetrate through clouds and can profile the atmosphere with a higher vertical resolution and accuracy, making it ideal for profiling the MBL.

The limitations in the use of GPS RO within the PBL region are primarily due to the existence of very large gradients of refractivity in the atmosphere. When these large vertical gradients of refractivity occur (known as super-refraction or ducting conditions), rays with tangent points inside the super-refraction (SR) layer are trapped in the duct. Rays that enter and leave the atmosphere might cross a SR layer, but they do not have their tangent point inside the layer (Sokolovskiy, 2003). SR conditions occur quite often over the western coasts of major continents in the subtropical ocean and trade wind regions (Xie et al., 2010). The inability to use these lower observations also limits our understanding of the processes that govern the climate, since the MBL is a very important component of the climate system, particularly 
a)

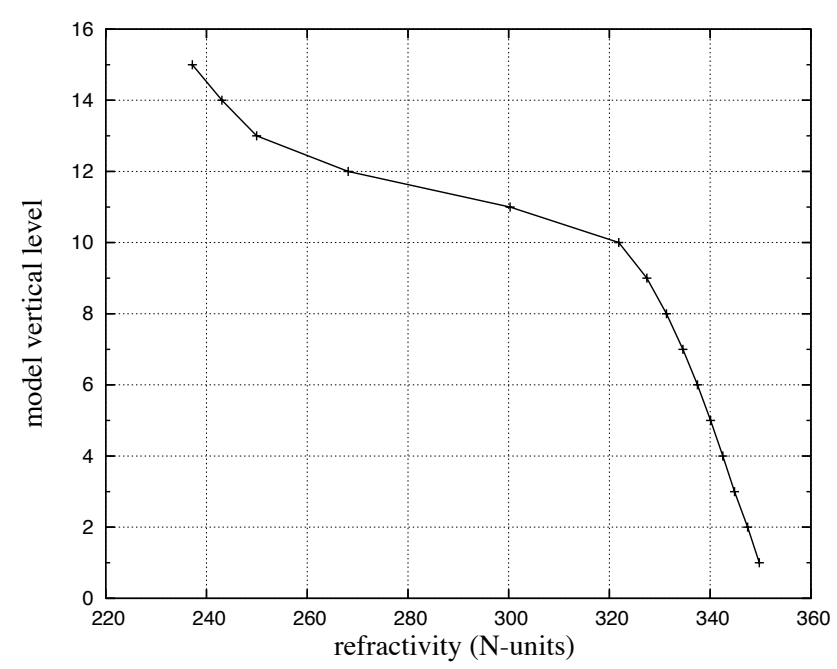

c)

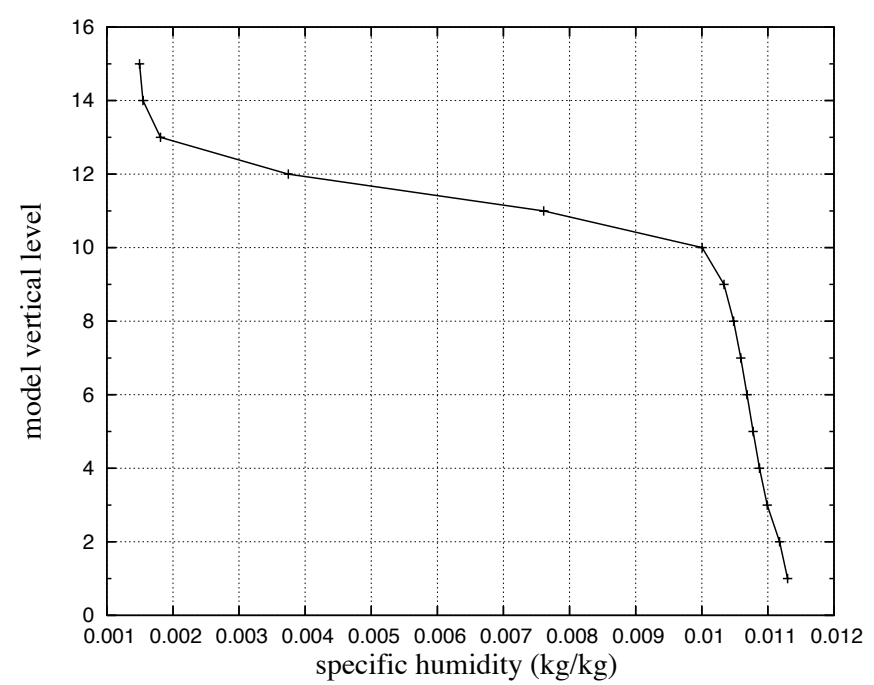

b)

d)
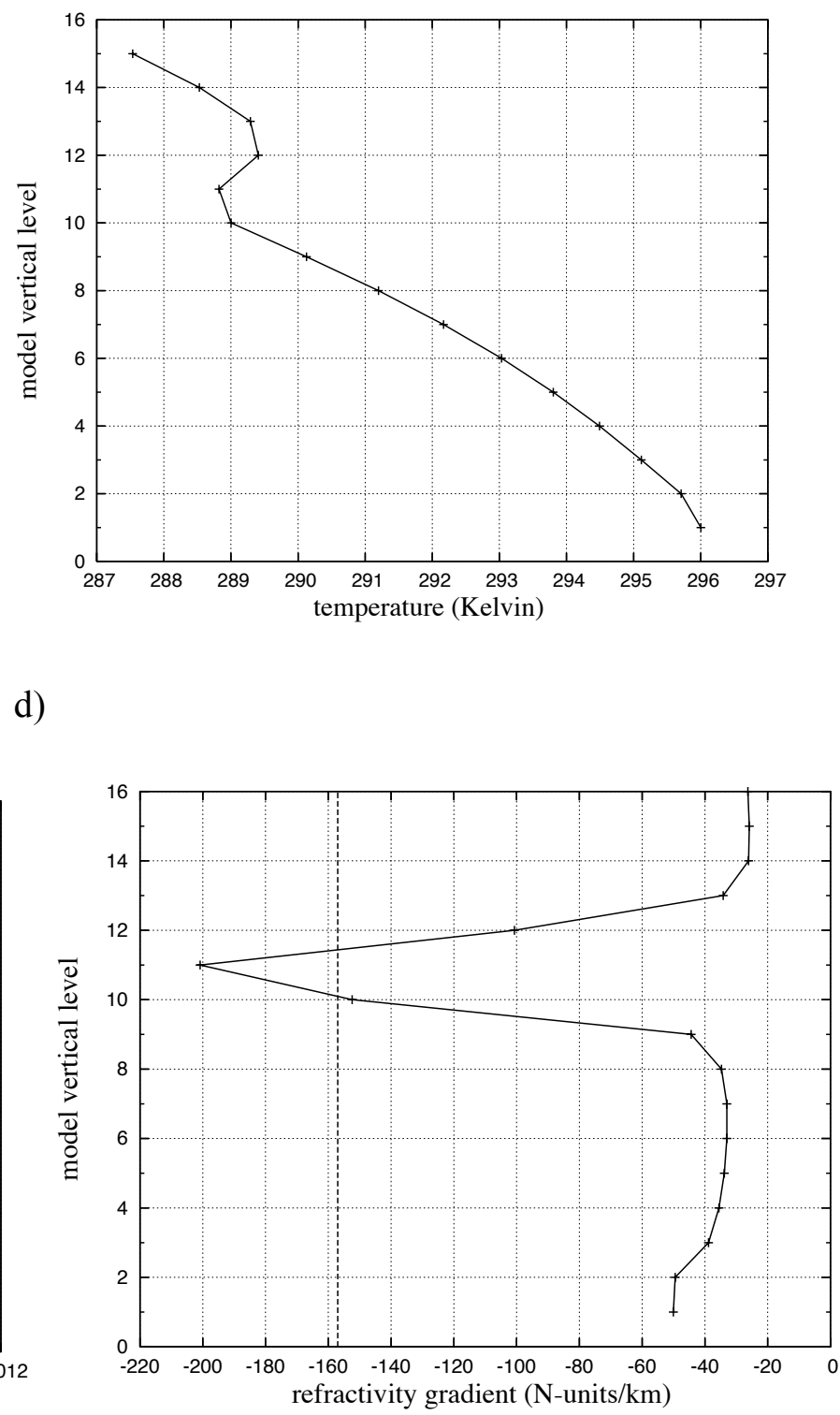

Figure 1. (a)-(d) Model vertical structure for a one-layer super-refraction case. The dashed vertical line in (d) indicates critical gradient.

in the trade wind region. SR is expected to occur frequently near the top of the boundary layer over oceans, as indicated in numerical weather prediction model analyses (von Engeln and Teixeira, 2004) and balloon soundings. As an example, Fig. 1 shows the gradient of refractivity for a case where the National Centers for Environmental Prediction (NCEP) model detected atmospheric SR conditions. Sometimes, the vertical gradient of refractivity exceeding the critical gradient (i.e., the value of the gradient of refractivity in the atmosphere that results in SR conditions, $-157 \mathrm{~N}$ units $\mathrm{km}^{-1}$ ) might extend to two model layers. In either case, a well-defined boundary layer is capped by a strong temper- ature inversion (Fig. 1b) and sharp negative moisture gradient (Fig. 1c). A study of the frequency and distribution of SR events at the European Centre for Medium-Range Weather Forecasts (ECWMF) was conducted by von Engeln and Nedoluha (2003) with the use of simulated RO measurements.

Under SR conditions, the assimilation of GPS RO below the height of the SR layer is an ill-conditioned problem: there are an infinite number of atmospheric states that would reproduce exactly the same GPS RO profile (Xie et al., 2006). When bending angle profiles are inverted into refractivities at the processing centers (Hajj et al., 1994; Kuo et al., 2004), 

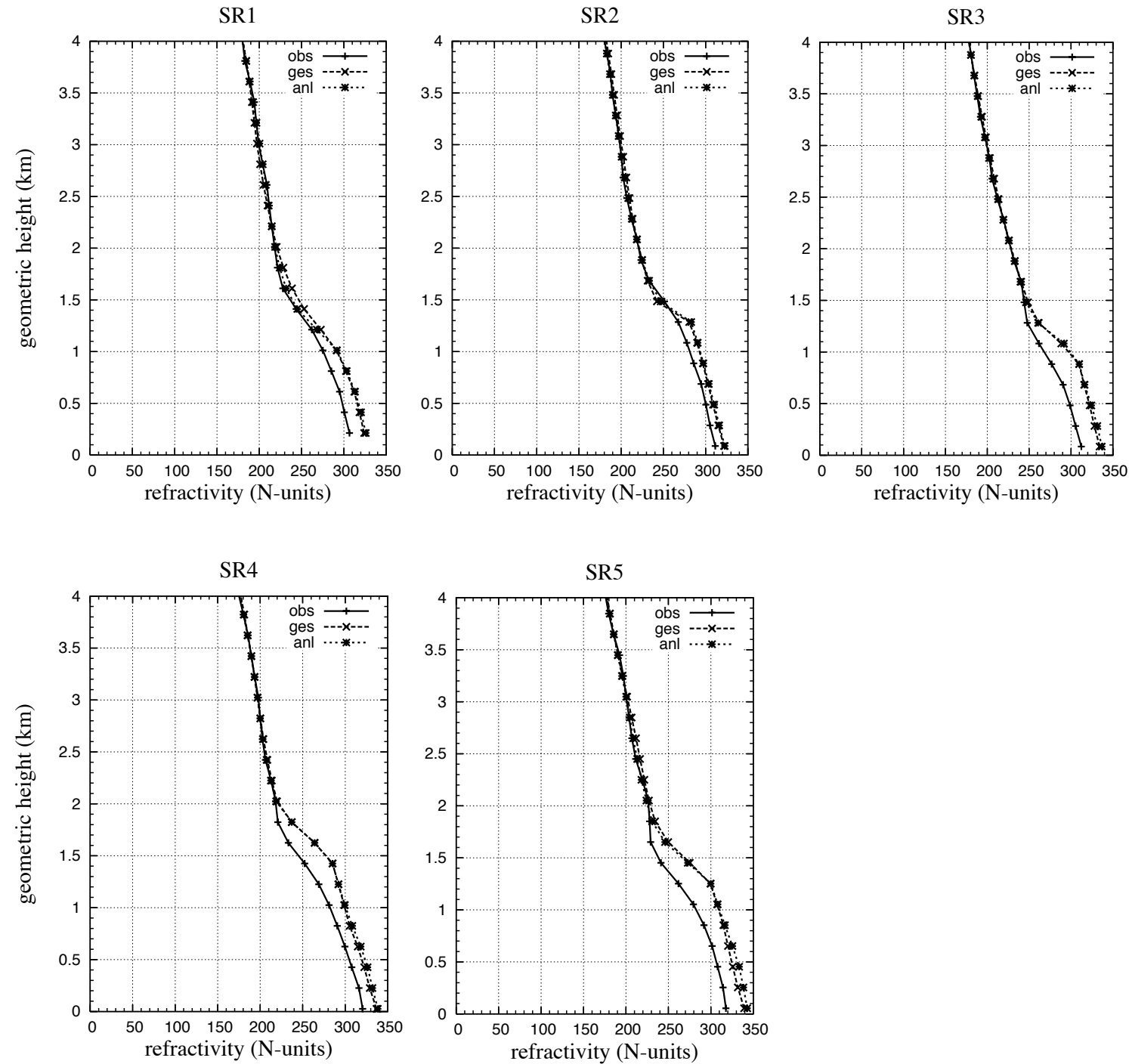

Figure 2. Refractivity as a function of the geometric height for five profiles likely affected by super-refraction conditions. Observation (obs) values as well as model background (ges) and analysis (anl) simulations are shown for each profile.

one of the possible solutions is retrieved, namely the one that has the lowest refractivity value. Therefore, refractivity observations are negatively biased under SR conditions at and below the height of the SR layer. In this case, observations need to be rejected in the assimilation algorithms. On the other hand, bending angles still contain the indetermination; thus observations might be rejected in a data assimilation system. However, other challenges exist when attempting to use these observations in weather models. For example, these low-level observations have a larger signal-to-noise ratio, and an infinite number of atmosphere states would reproduce exactly the same retrieved bending angle profile.

Work to evaluate ways to assimilate bending angles under the presence of SR conditions is currently under investigation at NOAA. This includes a modification of the current bending angle forward operator (Cucurull et al., 2013) and a reevaluation of the observation error characterization. Until this work is completed, the rejection of bending angles that might have been affected by SR conditions is necessary.

Quality controls aimed to identify profiles affected by SR conditions have been implemented at other operational centers. For example, observations located below regions where the refractivity lapse rate is below $-50 \mathrm{~km}^{-1}$, either from the observations or the background field, are rejected at Météo France (Poli et al., 2009). At the German Weather Service (Deutscher Wetterdienst), starting with an impact height of $8 \mathrm{~km}$, a profile section below a non-monotonous bending angle profile is discarded when the bending angle decreases by more than 3 times the assigned observation standard deviation (Anlauf et al., 2011). Bending angle observations are rejected at the European Centre for Medium-Range Forecasts when the refractivity gradient reaches half the critical 

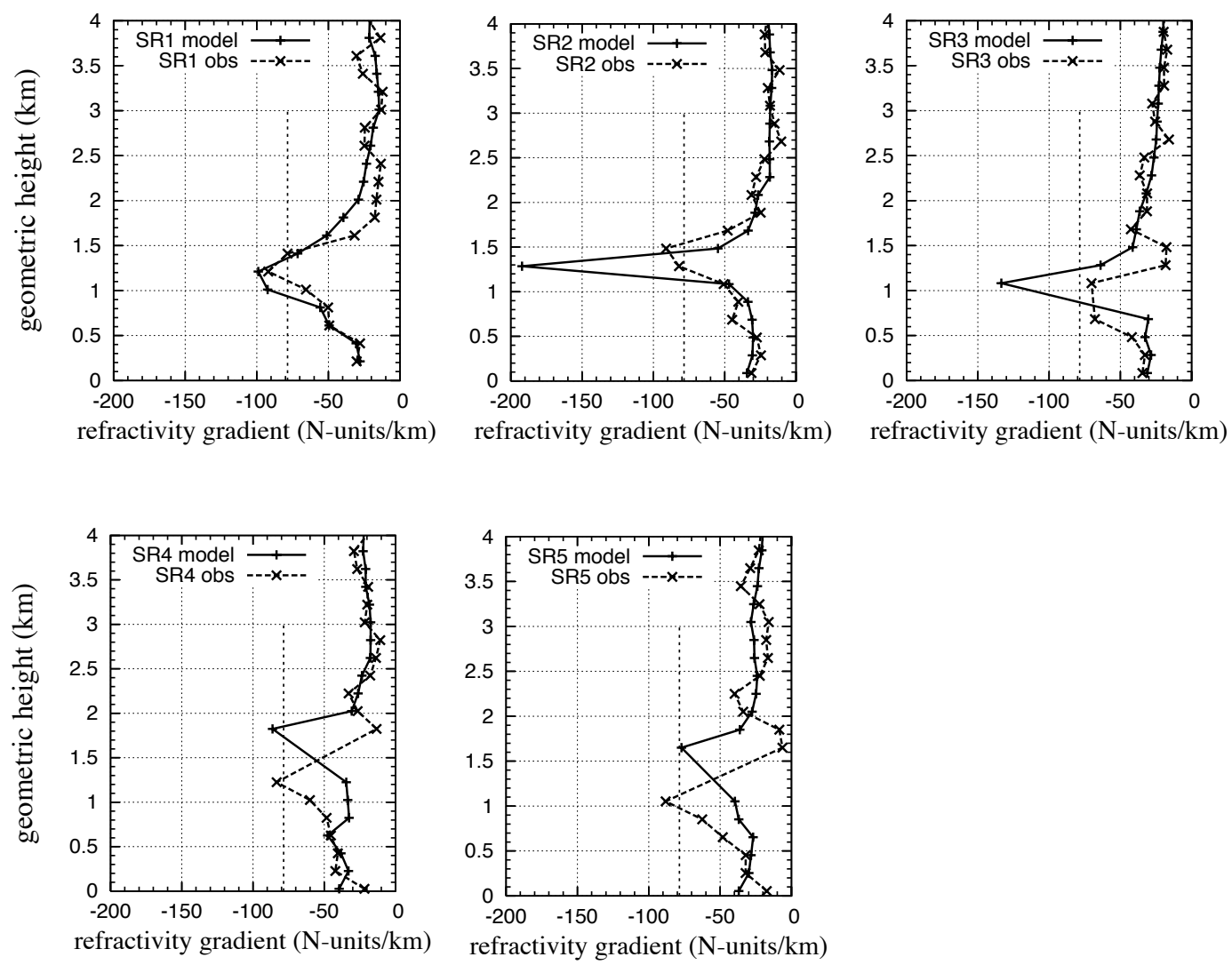

Figure 3. Model-simulated and observed refractivity gradient as a function of the geometric height for the five super-refraction profiles shown in Fig. 2. Half the critical gradient value is shown as a dashed line in each profile.
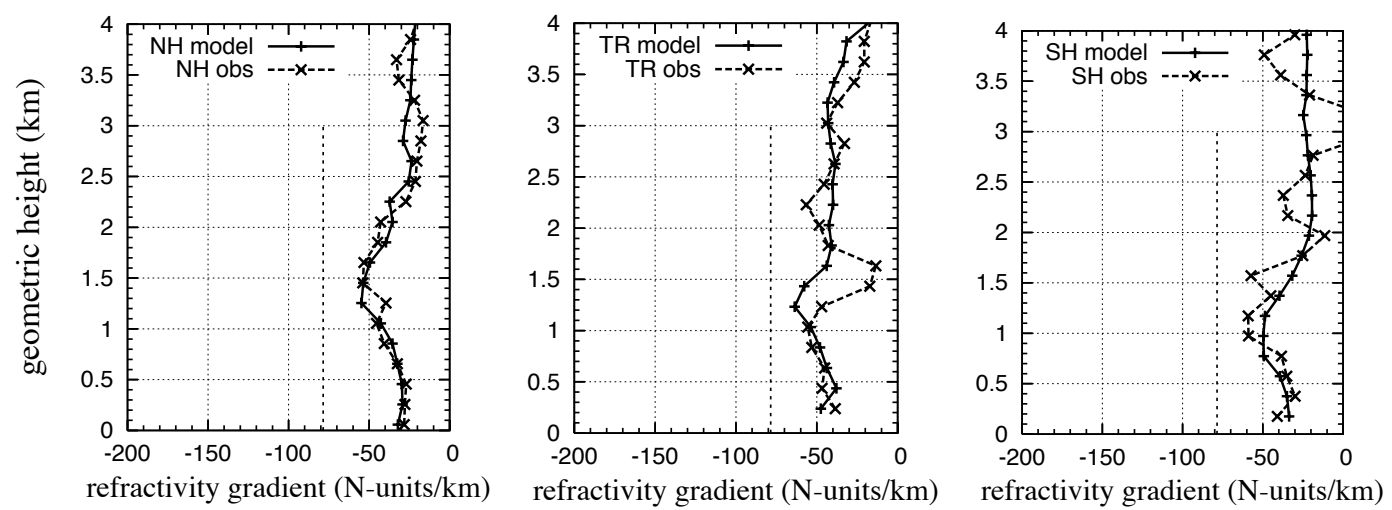

Figure 4. Model-simulated and observed refractivity gradient as a function of the geometric height for three standard profiles. Half the critical gradient value is shown as a dashed line in each profile.

gradient (S. B. Healy, personal communication, 2011). In this paper, we describe the implementation of specific quality control procedures within NCEP's global data assimilation system to detect and reject observations likely affected by SR conditions. Since NCEP's system can assimilate soundings of either refractivity or bending angle, algorithms for both types of retrievals have been implemented. Furthermore, the existence of SR atmospheric conditions, identified by the model and/or the observation profiles, is considered. This is the first time that NCEP has implemented quality control for observations under such atmospheric conditions.

The paper is organized as follows: refractivity and bending angle profiles likely affected by SR conditions are compared to profiles not affected by these atmospheric conditions in Sect. 2. Then, the implementation of quality controls to detect and reject RO observations affected by SR conditions is 
(a)

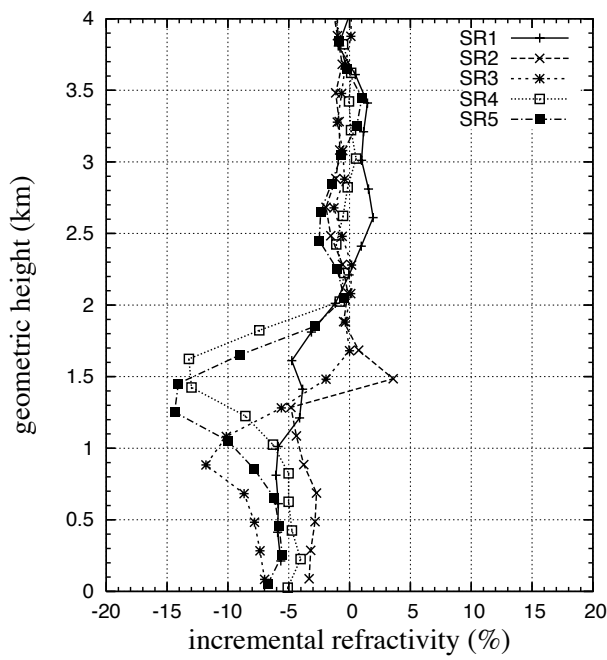

(c)

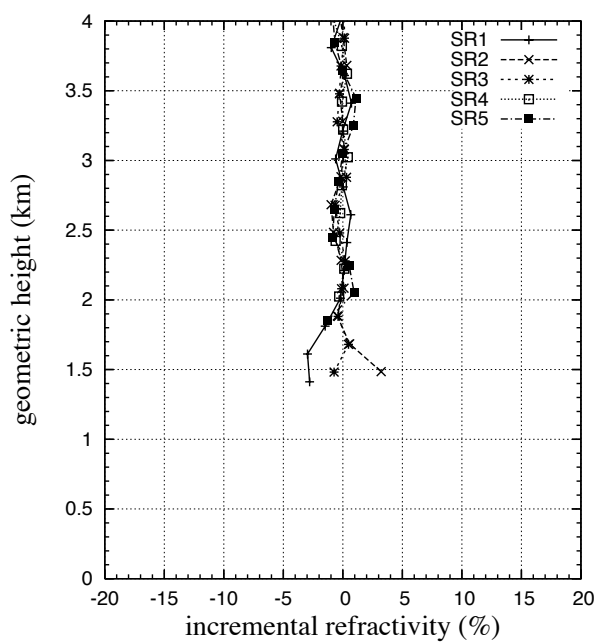

(b)

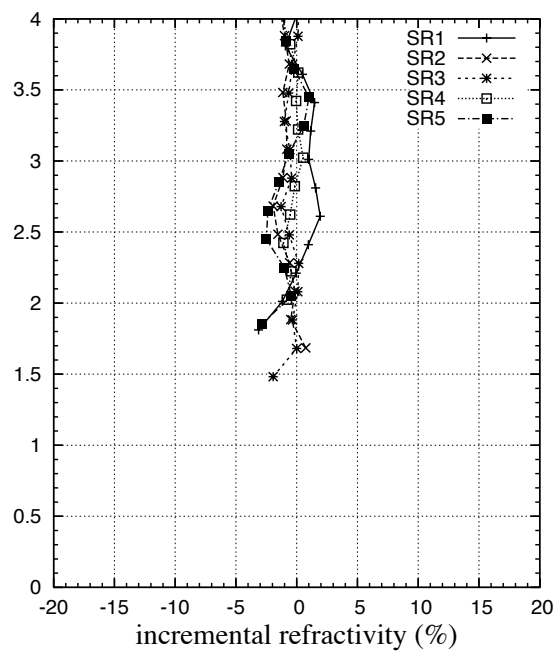

(d)

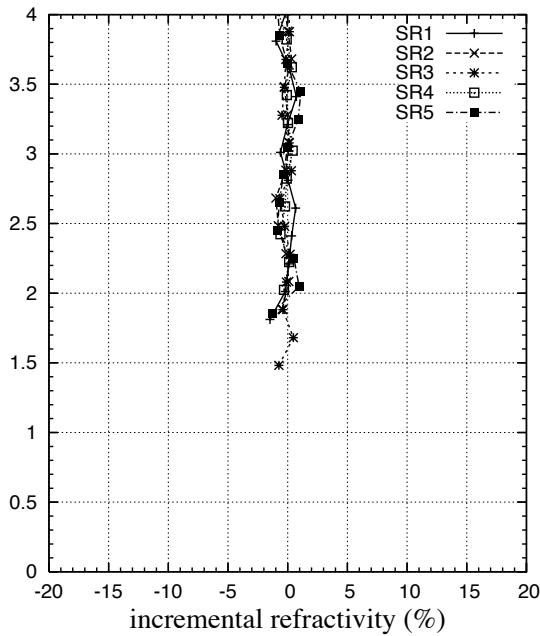

Figure 5. Differences between the observed and simulated refractivity profiles (in percentage) for (a) background field, (b) first outer iteration, (c) second outer iteration with the standard quality control, and (d) second outer iteration with the additional super-refraction quality control.

described in Sect. 3. Results from a forecast impact study with a simplified version of the operational NCEP's system are presented in Sect. 4. Finally, a summary is discussed in Sect. 5.

\section{Comparison of SR and non-SR profiles}

In this section, we compare profiles retrieved under standard atmospheric conditions against profiles likely affected by SR conditions. Profiles of both refractivity and bending angle are addressed, and the limitations of the current quality controls are described.

\subsection{Refractivity}

Refractivity profiles for five occultations identified as likely being affected by SR conditions are shown in Fig. 2. Profiles from the observations, background simulation, and analysis are represented in each figure. All five profiles are within the tropical latitudes. The negative bias starting at the top of the PBL is evident in all five profiles.

In order for the RO technology to be able to detect SR conditions, atmospheric layers need to extend $\sim 100 \mathrm{~m}$ in the vertical and $\sim 200 \mathrm{~km}$ in the horizontal (Kursinski et al., 1997). It is also important to take into account that the Abelretrieved refractivities cannot detect gradients of refractivity 
(a)

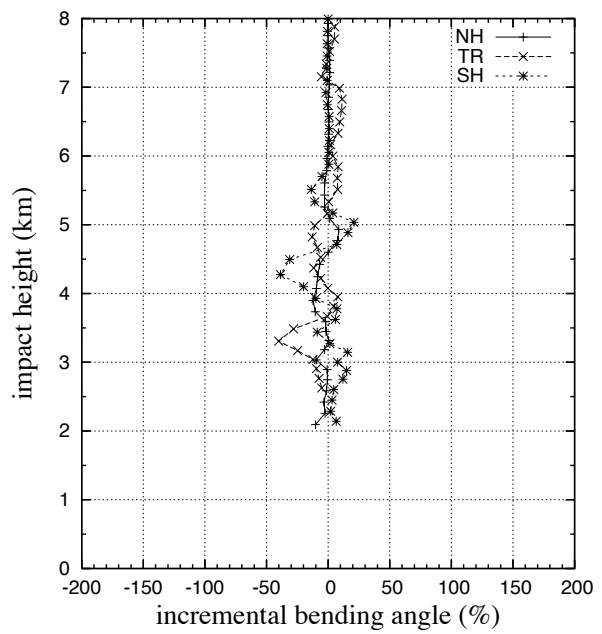

(b)

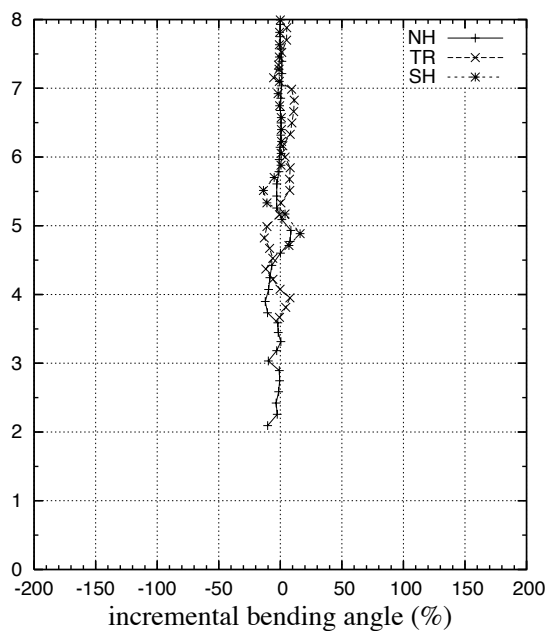

(c)

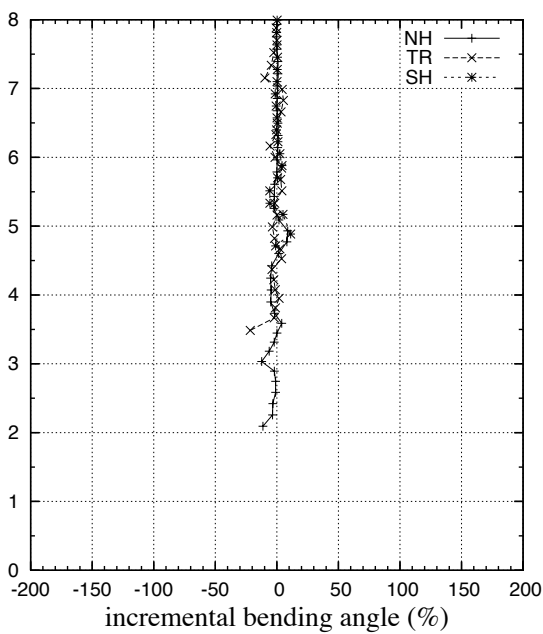

Figure 6. Differences between the observed and simulated bending angle profiles under standard atmospheric conditions (in percentage) for (a) background field, (b) first outer iteration, and (c) second outer iteration with the standard quality control.

(a)

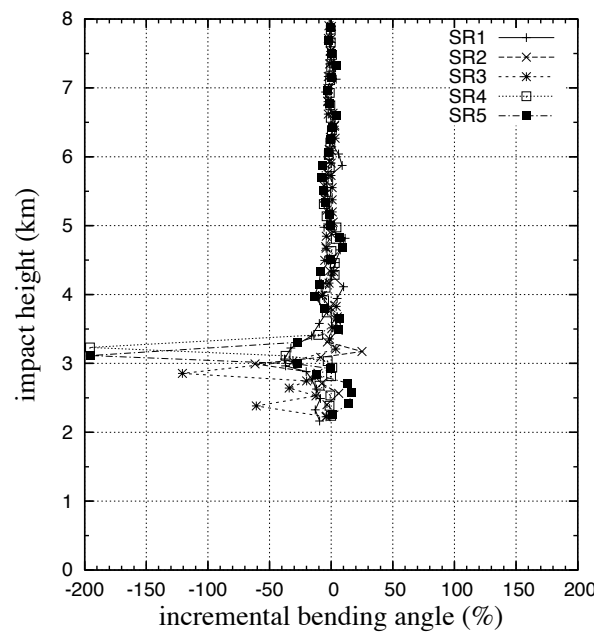

(b)

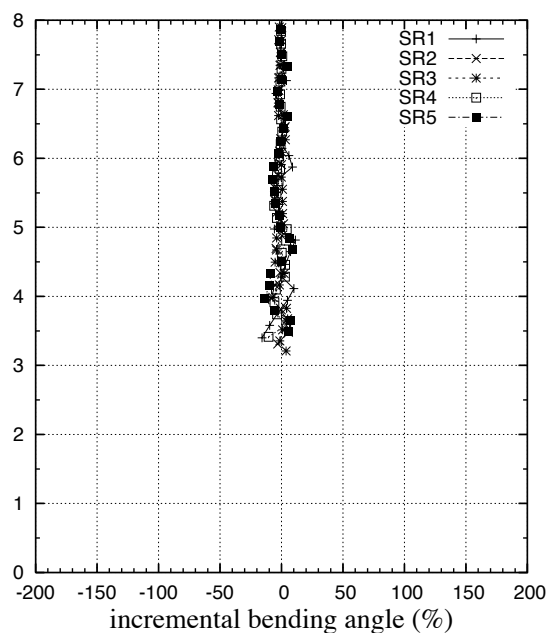

(c)

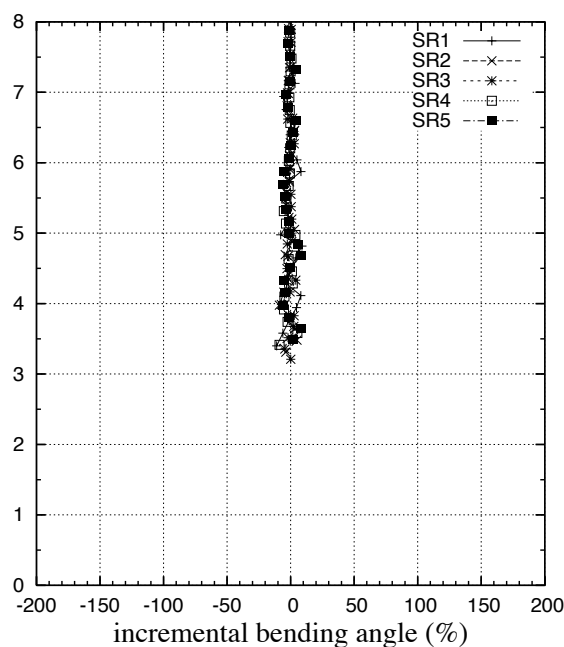

Figure 7. Differences between the observed and simulated bending angle profiles under super-refraction conditions (in percentage) for (a) background field, (b) first outer iteration, and (c) second outer iteration with the standard quality control.

exceeding or equal to the critical gradient value. It is unlikely we can even detect gradients close to this value because of the smoothing applied to bending angles processed with wave optics, which otherwise would be very noisy due to low signal-to-noise ratio in the lower troposphere and the effects of horizontal gradients. The conversion of high-resolution files to the lower vertical resolution Binary Universal Form for the Representation of meteorological data (BUFR) grid further smoothes the profiles, thus reducing the values of the refractivity gradient.
The model vertical resolution was found to adequately represent SR conditions in the lowest few kilometers as it ranges from $\sim 100$ to $250 \mathrm{~m}$ in the lowest $2 \mathrm{~km}$ and then increases to $\sim 500 \mathrm{~m}$ at $\sim 5 \mathrm{~km}$. This seems to indicate that the model should in principle at least be able to represent sharp bending angle structures in the lowest $2 \mathrm{~km}$. The refractivity gradients for the five profiles represented in Fig. 2 are shown in Fig. 3. Both gradients retrieved from the observation files, as well as from the model simulations, are represented. Note that in neither case is the sharp gradient of critical refraction 
SR1

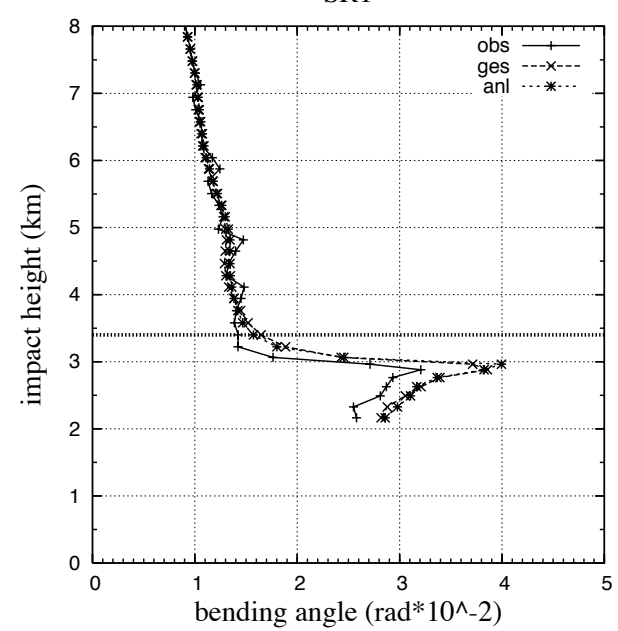

SR4

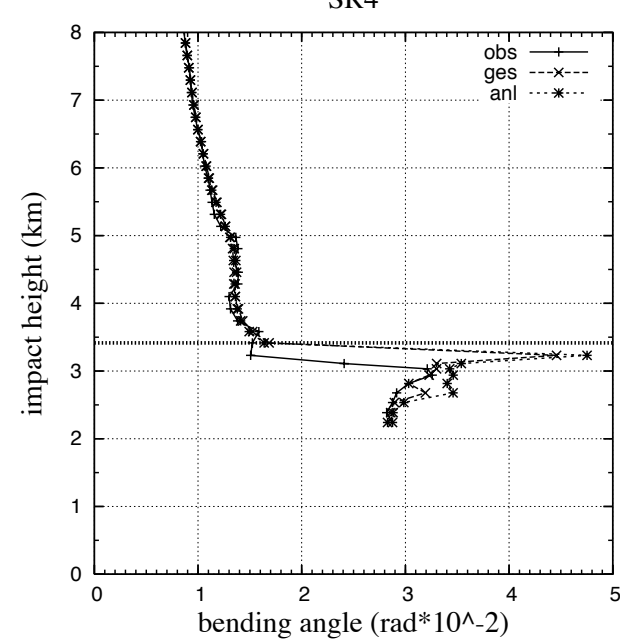

SR2
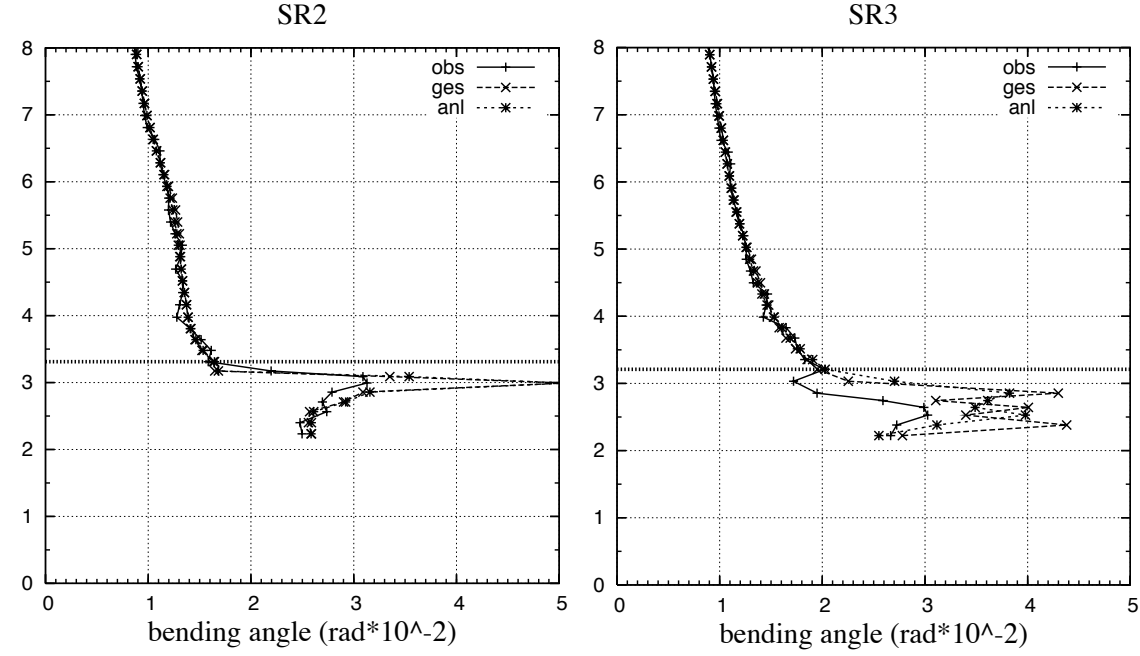

Figure 8. Model-simulated and observed bending angle profiles as a function of the impact height with the standard quality control. The dashed lines show the height of the lowest observation that passed the quality controls.

reached except for profile SR2, where the model detects and far exceeds this value. In general, the model seems to detect larger gradients than the observations. Also note that the largest gradient is not always found at the same height between observations and model simulations (e.g., profiles SR4 and SR5), likely indicating a mismatch in PBL height. It is expected that increasing the vertical resolution of the model will enable it to detect larger gradients of refractivity.

The vertical gradient of refractivity for three standard profiles (i.e., not affected by SR conditions) is shown in Fig. 4 . We selected one profile for each latitudinal range (Northern Hemisphere extratropics, Southern Hemisphere extratropics, and tropics). As expected, the values are significantly smaller than in the SR cases (Fig. 3).

The differences between the observations and model simulations (in percentage) for the five SR profiles identified in
Fig. 2 are shown in Fig. 5a. The negative values in the lower troposphere (reaching $\sim-15 \%$ in some cases) are another way to represent the negative bias in Fig. 2. The standard quality controls reject most of these negatively biased observations in the first outer iteration (Fig. 5b), but some observations that should have probably been removed made it into the assimilation algorithms (e.g., the lowest observations in profiles SR1, SR3, and SR5). In the second outer loop of the minimization process (Fig. 5c), some suspicious observations remain, and even additional observations from profiles SR1 and SR 2 now passed the standard quality controls. (The bias in the lower section of SR2 is positive rather than negative because the vertical gradient from the observation profile peaks at a higher altitude than the gradient from the model simulation, as seen in Fig. 3.) Thus, although most of the observations affected by SR conditions are already rejected by 
(a)

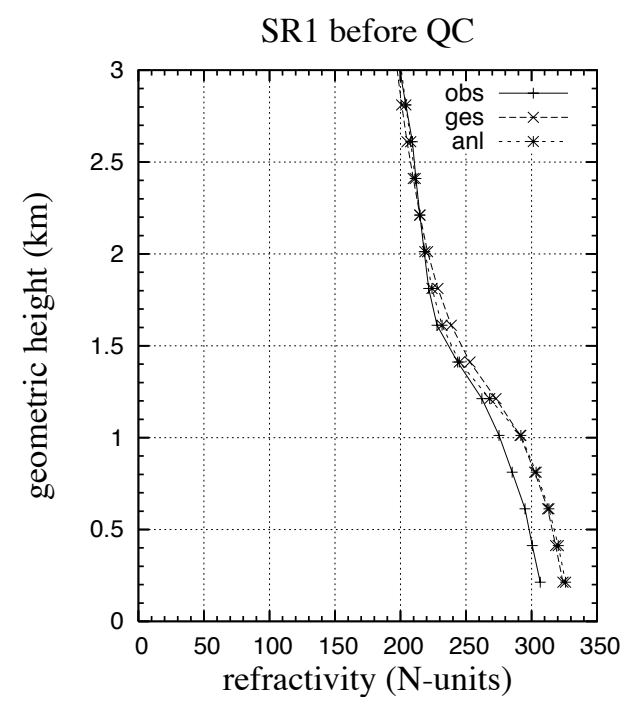

(b)

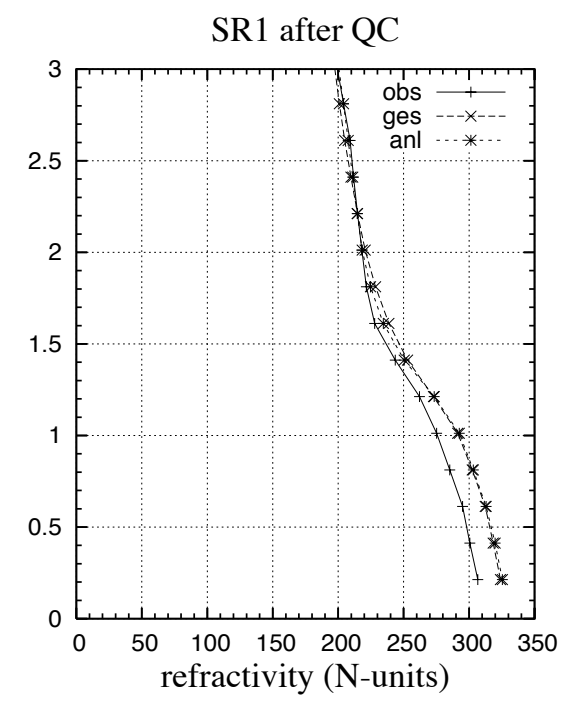

Figure 9. Observation, background, and analysis refractivity profiles as a function of the geometric height for (a) standard quality control and (b) super-refraction quality control cases.

the standard quality controls, some additional observations should have been removed from the assimilation system.

\subsection{Bending angle}

The differences in percentage between observations and model simulations for the three standard profiles used in Fig. 4 are shown in Fig. 6. Despite some values reaching $-50 \%$ in Fig. 6a, most of these outliers are removed with the standard quality controls in Fig. 6b. At the second outer loop (Fig. 6c), the differences are small, except perhaps for the lowest observation from the tropical profile. For the five SR profiles, although the standard quality controls also remove the outliers (Fig. $7 b$ and c), the differences between the observations and the background field (Fig. 7a) are significantly larger than for the profiles not affected by SR conditions. Note that in some cases the differences are as large as $-200 \%$. Observations showing these sharp biases with respect to the model simulation are rejected with the current quality controls. However, this is clearly not an optimal approach, because a mismatch between the modeled and the observed PBL height could result in very large differences between the observed and simulated values. When this occurs, observations, which might not necessarily be "bad", are rejected from the assimilation system. This situation is illustrated in Fig. 8, where the observed, simulated, and analyzed bending angles at the locations of the observations are plotted for the five SR profiles shown in Fig. 7a. For profiles SR4 and SR5, the spike in bending angle clearly takes place at a higher location in the model than in the observations. This results in the larger negative bias in bending angle seen in Fig. 7a for these two profiles. If the PBL height had been the same in the observations and model simulations, the differences would have been largely reduced and the observations would have likely passed the quality controls. The dashed horizontal lines in Fig. 8 indicate the height of the lowest observation that passed the standard quality controls. All the observations below this height are rejected in the assimilation algorithms.

\section{Updated quality controls}

In this section we describe the implemented SR quality controls for assimilating profiles of refractivity and bending angles.

\subsection{Refractivity}

The newly implemented SR quality control applies to observations at and below $3 \mathrm{~km}$ in geometric height. The model might not be able to detect SR atmospheric conditions above $\sim 2 \mathrm{~km}$ due to the limited vertical resolution, but this height is expected to rise as the model vertical resolution improves in the future. Observations are rejected if either the model or the observational gradient of refractivity reaches half the critical gradient. If this situation occurs, the rest of the profile below that observation is rejected as well.

With this quality control, we attempt to detect observations that might have been affected by SR conditions but have passed the standard checks. However, note that, if the model does not simulate a SR layer accurately (e.g., when the observation profile is far from reaching the critical value), the model could be creating unrealistic gradients and we would 
SR 1

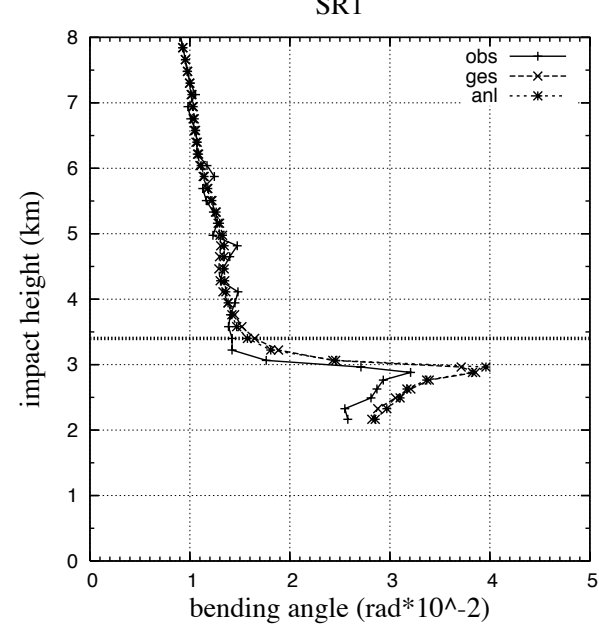

SR4

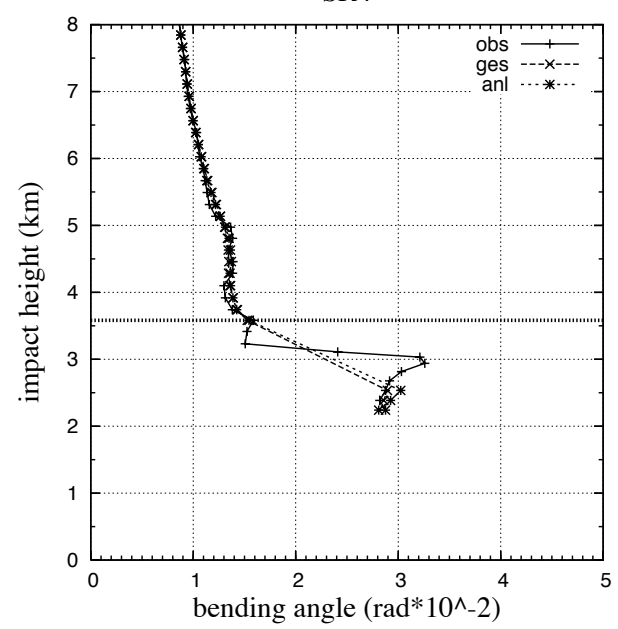

SR2

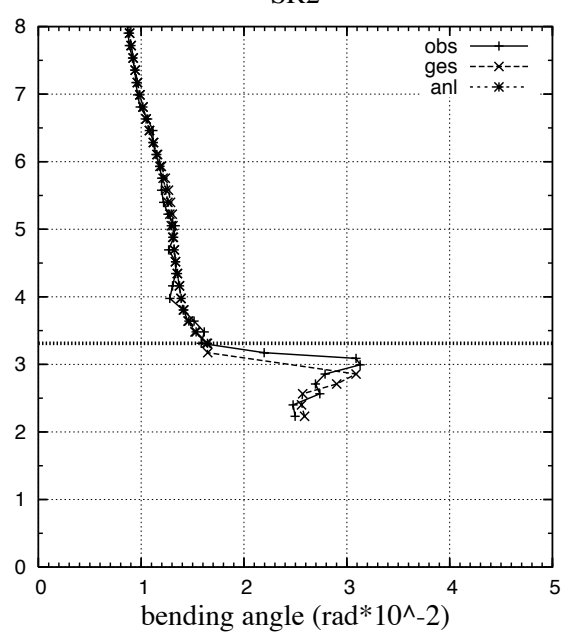

SR5

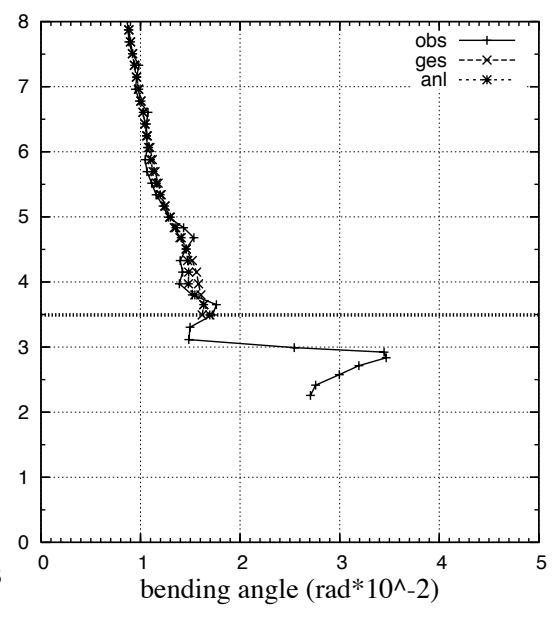

SR3

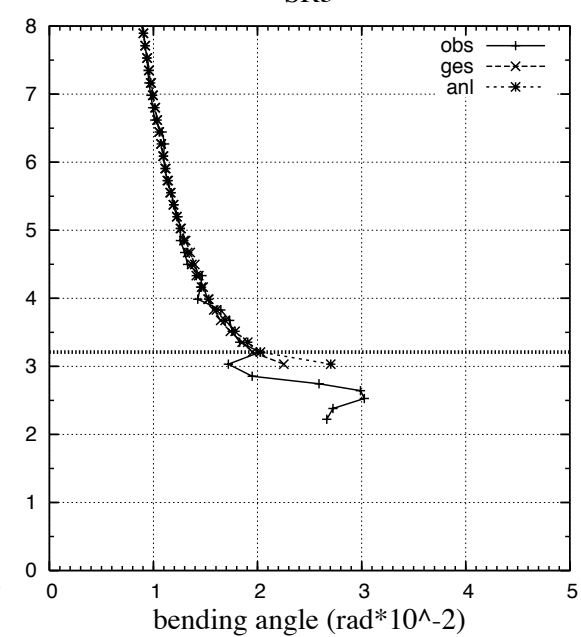

Figure 10. Model-simulated and observed bending angle profiles as a function of the impact height with the super-refraction quality control. The dashed lines show the height of the lowest observation that passed the quality controls.

be rejecting otherwise good observations. In the case where both the observations and the model reach half the critical gradient, and this situation occurs at a different height in the observations and the model, the quality control will use the observation with the highest geometric height.

Although most of the observations affected by SR conditions in Fig. 2 were already rejected by the standard quality controls, the impact of this new criterion can be seen in Fig. 5d. The lowest observation from profiles SR1 and SR2 that passed the standard quality controls (Fig. $5 \mathrm{c}$ ) is now rejected at the second outer loop (Fig. 5d). As a consequence, the analysis now tends towards the background filed at the heights of these rejected observations. This is illustrated in Fig. 9 for profile SR1. The analysis is closer to the background field at the geometric height of $\sim 1.5 \mathrm{~km}$ than it was before implementing the SR quality control. The lowest observations from profile SR3 in Fig. 5d passed the SR quality control because the gradient of refractivity for these observations is lower than half the critical gradient.

\subsection{Bending angle}

Under the SR and spherical symmetry approximation, the one-dimensional bending angle forward operator typically used at the operational weather centers formally approaches infinity as the tangent point of a ray within a profile reaches a SR layer (Sokolovskiy, 2003). Outside the SR layer, the simulated bending angle adopts a finite value again. However, the assimilation of observations below a SR is very challenging, and, until a methodology to make use of these observations can be tested, they need to be rejected from the assimilation system. SR can also occur on the observation side. It is important to note that from the observed profiles one cannot assure that SR occurred, but this might change with future RO constellations such as COSMIC-2 due to higher antenna 
gain (S. Sokolovskiy, personal communication, 2013). Both situations, i.e., SR from the model and SR from the observations, need to be addressed. As a consequence, the two following quality controls have been implemented.

On the model side, when $75 \%$ of the critical value is detected within a few model vertical layers surrounding the location of an observation, the observation, as well as the rest of the profile below this observation, is rejected. When several layers reach the lower limit of $75 \%$, the top layer is used. We did not use the exact value of the critical refraction. This was because we saw that, as the model gradient of refractivity approached the critical gradient, NCEP's bending angle forward operator became unstable, resulting in unrealistic simulated values in some cases. We found that a value of $75 \%$ was reasonable.

On the observation side, if a bending angle is larger than $0.03 \mathrm{rad}$ and the model detects at least $50 \%$ of the critical gradient within a few vertical layers surrounding the location of the observation, then from the observations that verify these two conditions we select the observation within the profile with the largest bending angle. Any observation within the same profile and below the selected observation is rejected, while we assimilate the section of the profile above.

The equivalent to Fig. 8, but using the new SR quality controls, is shown in Fig. 10. The height of the lowest observation that passed the updated quality controls is shown as a dashed line. Although the observation profiles are the same in Figs. 8 and 10, there are a few differences in the modelsimulated counterpart. In Fig. 8, model simulations for all the observations within the model vertical grid were provided in the background field and the analysis. With the updated quality controls, only observations within the model vertical grid that do not fail the model side SR quality control are used in the model simulations. This is because the bending angle is now only computed when an observation does not fail the model SR quality control. (Observations that fail the SR quality control on the model side do not have a bending angle model simulation counterpart.) From Figs. 8 and 10, the implementation of the SR quality control does not modify the rejection structure for profile SR1. For profile SR2, the large background and analysis-simulated values around $3 \mathrm{~km}$ are gone in Fig. 10. This situation corresponds to observations that fail the model SR check. In addition, there is no analysis counterpart in the lower troposphere, because all these observations fail the model SR quality control. However, despite the different reasons for rejection in profile SR2, the observations being rejected are the same with the SR and standard quality controls. A similar situation is found in profiles SR3 and SR5. (Note that the zig-zag structures in the Fig. 8 model simulations are gone in Fig. 10.) An additional observation is rejected with the SR quality control in profile SR4.
Table 1. Anomaly correlation score for the 6-day geopotential heights for the Northern $(\mathrm{NH})$ and Southern (SH) Hemisphere extratropics.

\begin{tabular}{lcccc}
\hline Experiment & $\begin{array}{c}\mathrm{NH} \\
(500 \mathrm{mb})\end{array}$ & $\begin{array}{c}\mathrm{NH} \\
(250 \mathrm{mb})\end{array}$ & $\begin{array}{c}\mathrm{SH} \\
(500 \mathrm{mb})\end{array}$ & $\begin{array}{c}\mathrm{SH} \\
(250 \mathrm{mb})\end{array}$ \\
\hline CTL & 0.751 & 0.770 & 0.766 & 0.796 \\
EXP & 0.755 & 0.771 & 0.767 & 0.798 \\
\hline
\end{tabular}

Table 2. Root-mean-squared errors for the 3-day tropical winds.

\begin{tabular}{lcc}
\hline Experiment & $\begin{array}{c}\text { rms winds } \\
\left(850 \mathrm{mb}^{-1} \mathrm{~m} \mathrm{~s}^{-1}\right)\end{array}$ & $\begin{array}{c}\text { rms winds } \\
\left(200 \mathrm{mb}^{-1} \mathrm{~m} \mathrm{~s}^{-1}\right)\end{array}$ \\
\hline CTL & 3.197 & 8.030 \\
EXP & 3.160 & 8.016 \\
\hline
\end{tabular}

\section{Forecast impact study}

We conducted an impact experiment during the period from 5 July through 9 August 2009. The impact study used a simplified version of the NCEP's operational configuration. A simplified configuration is typically used at NCEP to evaluate the impact of individual changes in the assimilation system. The parallel runs used bending angle profiles, as this is the observation type being used in the operational model. The control experiment (CTL) used the standard quality controls for the assimilation of RO, while the updated SR quality controls were applied in the experiment run (EXP).

Anomaly correlation scores for the 500 and $250 \mathrm{mb}$ geopotential heights at day 6 are shown in Table 1 for the Northern Hemisphere extratropics (latitudes above $20^{\circ} \mathrm{N}$ ) and Southern Hemisphere extratropics (latitudes below $20^{\circ} \mathrm{S}$ ). A slight improvement is found for all latitude ranges with the new SR quality controls. Tropical root-mean-squared error winds at day 3 are also improved with the updated quality controls (Table 2). Overall, a slight improvement is found for the different fields and vertical levels when the SR quality controls are used.

\section{Conclusions}

In preparation for the launch of COSMIC-2 in 2016, work is being developed at NOAA to improve the assimilation of $\mathrm{RO}$ observations in the lower troposphere, in particular in the tropical region and under SR conditions. In the meantime, an additional quality control to directly detect and reject observations that might have been affected by SR conditions (either in the model or in the retrieval process) has been implemented, and it became operational at NCEP in January 2015. In this paper, we have discussed the details of the implementation of these additional quality controls for the assimilation 
of refractivities and bending angles in NCEP's global data assimilation system.

It is important to emphasize that the SR quality controls described here are not intended to replace the existing quality control procedures for RO observations, but rather to detect and reject observations that might have passed the existing checks. Although most observations were already rejected by the existing quality controls, and the impact of these changes might be just slightly positive or neutral in a statistical sense, they can be significant in specific situations where the assimilation of bad observations might cause instabilities/errors in the analysis. We have shown cases where some observations have been rejected due to the SR quality controls.

The limitations of NCEP's bending angle forward operator under the presence of SR conditions is under current investigation, and evaluating the assimilation of observations that might have been affected by SR conditions will be addressed in a future study.

Acknowledgements. The author thanks Scott Hausman (former ESRL/Global Systems Division acting director) and Kevin Kelleher (current/ESRL Global Systems Division director) for funding this work. She also acknowledges Sergey Sokolovskiy for providing the five super-refraction profiles used in this study.

Edited by: U. Foelsche

\section{References}

Albrecht, B. A., Ramanathan, V., and Boville, B. A.: The effects of cumulus moisture transports on the simulation of climate with a general circulation model, J. Atmos. Sci., 43, 2443-2462, 1986.

Anlauf, H., Pingel, D., and Rhodin, A.: Assimilation of GPS radio occultation data at DWD, Atmos. Meas. Tech., 4, 1105-1113, doi:10.5194/amt-4-1105-2011, 2011.

Betts, A. K. and Ridgeway, W.: Climate equilibrium of the atmospheric convective boundary layer over the tropical ocean, J. Atmos. Sci., 46, 2621-2641, 1989.

Clarke, R. H. and Brook, R. R.: The Koorin Expedition - Atmospheric Boundary Layer Data over Tropical Savannah Land, Australian Government Publishing Service, 359 pp., 1979.

Cucurull, L., Derber, J. C., and Purser, R. J.: A bending angle forward operator for GPS Radio Occultation measurements, J. Geophys. Res., 118, 1-15, doi:10.1029/2012JD017782, 2013.
Hajj, G. A., Kursinski, E. R., Bertiger, W. L., Romans, L. J., and Hardy, K. R.: Assessment of GPS occultations for atmospheric profiling, Preprints, Seventh Conf. on Satellite Meteorology and Oceonography, Monterey, CA, Am. Meteorol. Soc., J7-J10, 1994.

Heckley, W. A.: Systematic errors in the ECWMF operational forecasting model tropical regions, Q. J. Roy. Meteorol. Soc., 111, 709-738, 1985.

Izumi, Y.: Kansas 1968 field program data report, Air Force Cambridge Research Laboratory Paper 379, AFCRL-72-0041, Bedford, MA, 79 pp., 1971.

Kuo, Y.-H., Wee, T.-K., Sokolovskiy, S., Rocken, C., Schreiner, W., Hunt, D., and Anthes, R. A.: Inversion and error estimation of GPS radio occultation data, J. Meteor. Soc. Japan, 82, 507-531, 2004.

Kursinski, E. R., Hajj, G. A., Hardy, K. R., Schofield, J. T., and Linfield, R.: Observing Earth's atmosphere with radio occultation measurements, J. Geophys. Res., 102, 23429-23465, 1997.

Lettau, H. H. and Davidson, B.: Exploring the Atmosphere's First Mile, Pergamon Press, 578 pp., 1957.

Poli, P., Moll, P., Puech, D., Rabier, F., and Healy, S.: Quality Control, Error Analysis, and Impact Assessment of FORMOSAT-3/COSMIC in Numerical Weather Prediction, Terr. Atmos. Ocean. Sci., 20, 101-113, doi:10.3319/TAO.2008.01.21.02(F3C), 2009.

Sokolovskiy, S: Effect of superrefraction on inversions of radio occultation signals in the lower troposphere, Radio Sci., 38, 1058, doi:10.1029/2002RS002728, 2003.

Swinbank, W. C.: A comparison between predictions of dimensional analysis for the constant flux layer and observations in unstable conditions, Q. J. Roy. Meteorol. Soc., 94, 460-467, 1968.

Von Engeln, A. and Nedoluha, G.: An analysis of the frequency and distribution of ducting events in simulated radio occultation measurements based on ECMWF fields, J. Geophys. Res., 108, 21,4669, doi:10.1029/2002JD003170, 2003.

Von Engeln, A. and Texeira, L.: A ducting climatology derived from the European Centre for Medium-Range Weather Forecasts global analysis fields, J. Geophys. Res., 109, D18104, doi:10.1029/2003JD004380, 2004.

Xie, F., Syndergaard, S., Kursinski, E. R., and Herman, B. M.: An approach for retrieving marine boundary layer refractivity from GPS occultation data in the presence of super-refraction, J. Atmos. Oceanic Technol., 23, 1629-1644, doi:10.1175/JTECH1996.1, 2006.

Xie, F., Wu, D. L., Ao, C. O., Kursinski, E. R., Mannucci, A. J., and Syndergaard, S.: Super-refraction effects on GPS radio occultation refractivity in marine boundary layers, Geophys. Res. Lett., 37, L11805, doi:10.1029/2010GL043299, 2010. 